\title{
Features of the Upgraded Imaging for Hypersonic Experimental Aeroheating Testing (IHEAT) Software
}

\author{
Michelle L. Mason ${ }^{1}$, Shann J. Rufer ${ }^{2}$ \\ NASA Langley Research Center, Hampton, VA, 23681
}

\begin{abstract}
The Imaging for Hypersonic Experimental Aeroheating Testing (IHEAT) software is used at the NASA Langley Research Center to analyze global aeroheating data on wind tunnel models tested in the Langley Aerothermodynamics Laboratory. One-dimensional, semi-infinite heating data derived from IHEAT are used in the design of thermal protection systems for hypersonic vehicles that are exposed to severe aeroheating loads, such as reentry vehicles during descent and landing procedures. This software program originally was written in the PV-WAVE ${ }^{\circledR}$ programming language to analyze phosphor thermography data from the two-color, relative-intensity system developed at Langley. To increase the efficiency, functionality, and reliability of IHEAT, the program was migrated to MATLAB ${ }^{\circledR}$ syntax and compiled as a stand-alone executable file labeled version 4.0. New features of IHEAT 4.0 include the options to perform diagnostic checks of the accuracy of the acquired data during a wind tunnel test, to extract data along a specified multi-segment line following a feature such as a leading edge or a streamline, and to batch process all of the temporal frame data from a wind tunnel run. Results from IHEAT 4.0 were compared on a pixel level to the output images from the legacy software to validate the program. The absolute differences between the heat transfer data output from the two programs were on the order of $10^{-5}$ to $10^{-7}$. IHEAT 4.0 replaces the PV-WAVE ${ }^{\circledR}$ version as the production software for aeroheating experiments conducted in the hypersonic facilities at NASA Langley.
\end{abstract}

\section{Nomenclature}

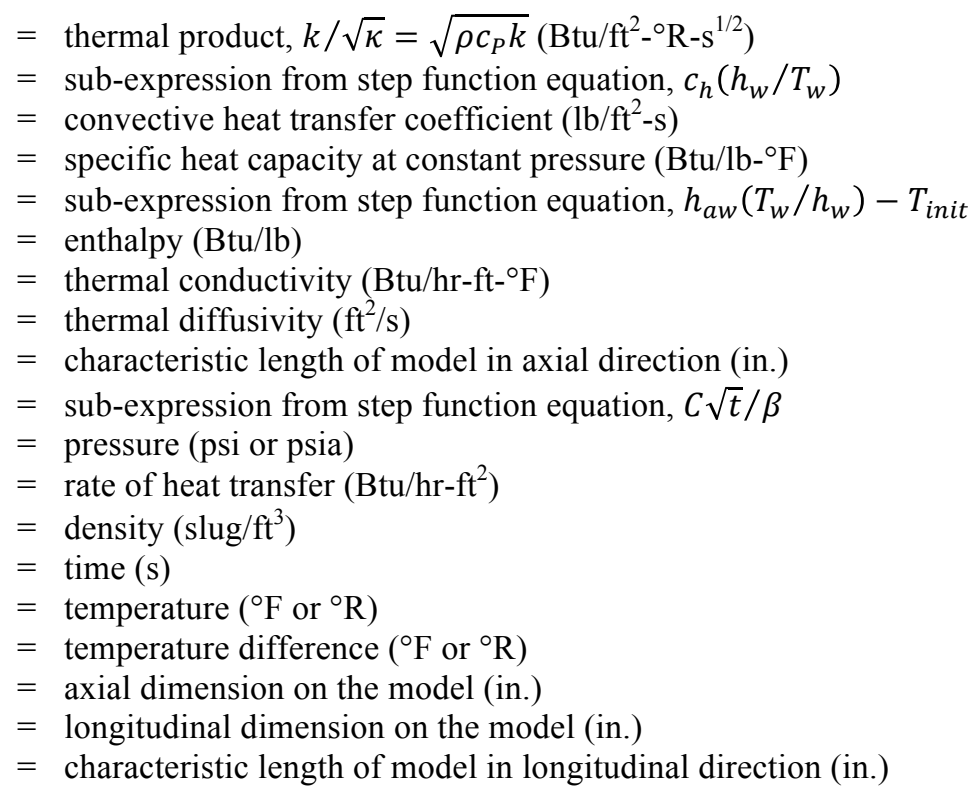

${ }^{1}$ Aerospace Engineer, Aerothermodynamics Branch, MS 408A, AIAA Member

2 Aerospace Engineer, Aerothermodynamics Branch, MS 408A, AIAA Associate Fellow 


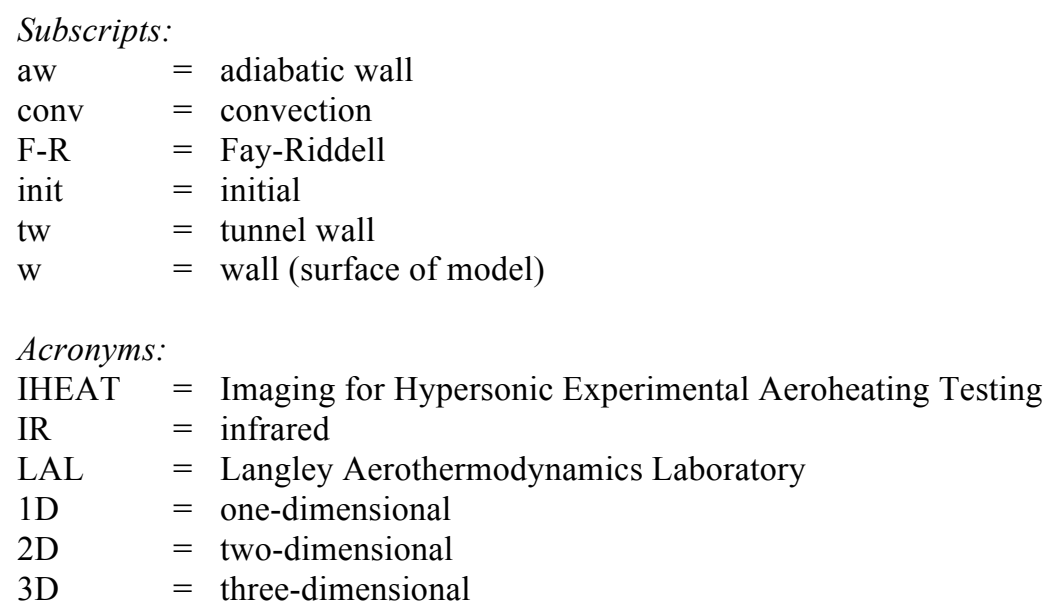

\section{Introduction}

$\mathrm{G}^{1,}$ lobal phosphor thermography is utilized in the NASA Langley Aerothermodynamics Laboratory (LAL) hypersonic facilities to predict aeroheating loads on aerospace vehicles. The Imaging for Hypersonic Experimental Aeroheating Testing (IHEAT) software program is used to analyze the phosphor thermography data obtained from scaled ceramic models tested in the LAL wind tunnels, which include the 15-Inch Mach 6 High Temperature, the 20-Inch Mach 6, and the 31-Inch Mach 10 Tunnels. The original IHEAT program was developed in the early 1990s and has served as the production-level program to analyze aeroheating data in the LAL facilities since that time. ${ }^{1,2}$ The latest version of the software, IHEAT version 3.2, recently was migrated from the PVWAVE $^{\circledR}$ programming language to MATLAB ${ }^{\circledR}$ and compiled as a stand-alone executable file called IHEAT 4.0. As an executable file, IHEAT 4.0 is resilient to changes to the base MATLAB ${ }^{\circledR}$ programming language. Thus, switching to MATLAB ${ }^{\circledR}$ syntax and adding new features to the 4.0 version of IHEAT improved the efficiency, functionality, and reliability of the program. These new tools simplify the data reduction process and reduce the time required to analyze the phosphor thermography data. Several manual data analysis steps were automated to increase the ease of use of the program. IHEAT 4.0 is run on a separate Unix server, accessed remotely through the researcher's host system, which ensures the program has sufficient memory to complete complex tasks without slowing down the host computer. The output data from IHEAT 4.0 were compared to results from IHEAT 3.2 to verify the algorithm in the upgraded program matches the process in the legacy software to compute heat transfer coefficients from wind tunnel data for the LAL facilities. The IHEAT program upgrade is one part of several modifications and calibrations that recently have been performed to improve the capabilities of and the data acquired from the LAL facilities. ${ }^{3}$ Examples of previous studies that used the phosphor thermography capability are provided in Refs. 4-20.

\section{Facilities}

The LAL consists of three conventional hypersonic blow-down facilities that are utilized for aerodynamic and aerothermodynamic tests as well as for basic fluid dynamic studies. Each of these facilities uses heated, dried, and filtered air as the test gas and provides for a variety of free-stream Reynolds numbers at free-stream Mach numbers of either 6 or 10. Berger et al. ${ }^{3}$ provides a detailed description of these facilities.

\section{A. 20-Inch Mach 6 Tunnel}

Typical operating conditions for the 20-Inch Mach 6 Tunnel are stagnation pressures ranging from 30 to 475 psia, stagnation temperatures from $380^{\circ} \mathrm{F}$ to $475^{\circ} \mathrm{F}$, and free-stream unit Reynolds numbers from 0.5 to 8 million per foot. A two-dimensional (2D), contoured nozzle is used to provide nominal free-stream Mach numbers from 5.8 to 6.1. The test section is 20.5 by 20 inches; the nozzle throat is 0.34 by 20 inches. The maximum run time is 20 minutes when a steam ejector attached to the vacuum system is used. Heating tests generally have total run times of less than 30 seconds, with an actual model residence time on the tunnel centerline of approximately 5-10 seconds.

Models are mounted on the injection system located in a housing below the closed test section. The injection carriage is raised mechanically through the use of a hydraulic pump, accumulator, and injection cylinder. The strut is attached to the hydraulic ram, which is supported in the cylinder through the use of roller bearings. When adjusting yaw, the ram and the strut are rotated within the cylinder. The computer-operated model control system is capable of 
moving the model through a pitch angle range of $-5^{\circ}$ to $+50^{\circ}$ and yaw angles of $\pm 10^{\circ}$. The facility core size is approximately 12-14 inches across, depending on Reynolds number.

The facility has six optical access ports, two on each side and two on the top of the test section. The front set of top and side windows are located at the model center of rotation point and are made of Corning 7940, Grade 5F, schlieren-quality glass. The top front optical access point can be fitted with a special zinc selenide (ZnSe) antireflective-coated window for use during infrared (IR) emission testing. The aft set of top and side windows are located behind the model center of rotation.

\section{B. 31-Inch Mach 10 Tunnel}

Typical operating conditions for the 31-Inch Mach 10 Tunnel are stagnation pressures ranging from 350 to 1450 psia, a stagnation temperature of approximately $1325^{\circ} \mathrm{F}$, and free-stream unit Reynolds numbers from 0.5 to 2 million per foot. A three-dimensional (3D), contoured nozzle is used to provide nominal free-stream Mach numbers from 9.6 to 10.0. The test section is 31 inches square; the nozzle throat is 1.07 inches.

Models are supported on a hydraulically-operated, sidewall-mounted injection system. The angle of attack can be varied from -90 to +90 degrees and the tunnel has a 14-in. inviscid core. Sideslip range is \pm 5 deg. With the second minimum closed to about 25 percent of the maximum test section cross section area and the use of two $41-\mathrm{ft}$ diameter and one $60-\mathrm{ft}$ diameter vacuum spheres, run times up to 120 seconds can be achieved. Heating tests generally have total run times of less than 30 seconds, with an actual model residence time on the tunnel centerline of approximately $5-10$ seconds.

The facility has three rectangular optical access ports on the top, bottom, and one side of the test section. The windows are centered at the model center of rotation and are made of schlieren-quality glass. The top access point can be fitted with a special $\mathrm{ZnSe}$ antireflective-coated window for use during IR testing.

\section{15-Inch Mach 6 Tunnel}

The 15-Inch Mach 6 Tunnel has stagnation pressures ranging from 100 to 400 psia, stagnation temperatures ranging from $400^{\circ} \mathrm{F}$ to $750^{\circ} \mathrm{F}$, free-stream unit Reynolds numbers from 1 to 7 million per foot, and nominal free-stream Mach numbers from 5.9 to 6 . The tunnel has a walk-in open-jet test section and an axisymmetric contoured nozzle with a throat diameter of $1.81 \mathrm{in}$., a nozzle exit diameter of $14.57 \mathrm{in}$., and a length of $75.6 \mathrm{in}$. The hydraulically-driven injection and retraction support mechanism can be used to vary the angle of attack from $-10^{\circ}$ to $50^{\circ}$ and the sideslip angle between $\pm 10^{\circ}$. The vacuum system for this facility is shared with the 31-Inch Mach 10 Tunnel.

The tunnel is equipped with numerous optical access ports, including three $29 \mathrm{in.}$ x $23 \mathrm{in}$. windows, located on each side and on the top of the tunnel, and four 5.5-in. diameter circular windows (two on each side) located approximately $45^{\circ}$ above the model. The maximum run time for this facility is 90 seconds utilizing all vacuum spheres and pumps.

\section{Phosphor Thermography System}

The two-color relative-intensity phosphor thermography measurement technique is used to obtain global experimental aeroheating data in the LAL facilities. This technique uses a mixture of phosphors that, when illuminated with ultraviolet light, fluoresce in the red and green portions of the visible spectrum. The intensity of the fluorescence is dependent upon the amount of incident ultraviolet light and the local surface temperature of the phosphor. This phosphor mixture, which is suspended in a silica ceramic binder and applied with an air brush, is used to coat the surface of a slip-cast silica ceramic wind tunnel model. The final coating thickness is approximately 0.001 in. Fiducial reference marks are then applied to the models using Dykem ${ }^{\circledR}$ blue ink to correlate distances between the marks to the distances between the pixels in 2D images of the model.

Using a 3-Charge Coupled Device (3-CCD) camera, fluorescence intensity images of an illuminated phosphor model exposed to the heated hypersonic flow of the tunnel are acquired and converted to temperature mappings via a temperature-intensity calibration. ${ }^{3}$ This calibration, which is derived for each new batch of the phosphor mixture, uses the red and green components of the image to construct a lookup table that converts the intensities to a temperature value. Currently, this calibration is valid over a temperature range from $65^{\circ} \mathrm{F}$ to $320^{\circ} \mathrm{F}$. The temperature data from the time-sequenced images taken during the wind tunnel run are then reduced to enthalpy-based heat transfer coefficients at every pixel on the image (and hence globally on the model) using the production-level software program referred to as IHEAT.

This technique offers four main advantages over conventional transient thin-skin calorimeter and thin-film resistance methods. The first is the global resolution of temperature and heating data that provides detailed information of specific flow features. Surface heating is determined in a global sense; data are computed at every point on the model surface within the field of view of the camera and at various times during the run. Secondly, the 
cast fused-silica models are relatively inexpensive to manufacture compared to machined models such as Macor ${ }^{\circledR}$ or metal models. Thirdly, the fabrication method used to build these ceramic models produces a coating that does not require re-application between runs, thereby significantly enhancing the efficiency of the phosphor technique. Finally, the slip-casting method is a rapid process whereby, in three to four weeks, a full array of inexpensive models can be fabricated, complete with various perturbations needed for a configuration build-up scheme such as variable nose radii and control flap deflections.

The IHEAT 4.0 program assumes one-dimensional (1D) heat conduction through a semi-infinite ceramic wind tunnel model with a convective boundary condition at the windward surface. Convective heat transfer coefficients, $c_{h}$, are calculated from a convective heat transfer, $\dot{q}_{c o n v}$, equation based on an enthalpy difference, i.e.,

$$
\dot{q}_{c o n v}=c_{h}\left(h_{a w}-h_{w}\right)
$$

The assumption that the model is semi-infinite in the direction normal to the model surface is reasonable since data are acquired after the model is exposed to the flow for, at most, a few seconds, so heat applied at the surface does not pass through the thickness of the ceramic shell during the short wind tunnel run. Multidimensional conduction analyses are necessary to improve the accuracy of the heating profile for data with sharp temperature gradients (for example, in the region near a shock-shock interaction) or near thin geometrical features such as leading edges and certain control surfaces. ${ }^{21}$

As the model is injected into the wind tunnel, the model passes through the tunnel boundary layer and then enters the tunnel core flow at the specified flow conditions. To account for the difference in heating between the tunnel centerline and the boundary layer, a step function represents the heat added to the model as the model passes through the boundary layer. Equation (2) shows this step function, which is computed using Laplace transforms:

$$
\frac{\theta_{w}}{D}=1-e^{\Lambda^{2}} \operatorname{erfc\Lambda }
$$

where the variable $\theta_{w}$ is defined as the difference between the surface temperature of the model at a specified time during the run, $T_{w}$, and the initial temperature of the model surface, $T_{i n i t}$, as shown in Eq. (3). Therefore, only the initial temperatures and the temperatures at some subsequent time during the run are needed to compute heat transfer coefficients $\left(c_{h}\right)$ at the surface of the model at that instant $(t)$. The variable $\Lambda$ is defined in Eq. (4) in terms of the run time $t$, the thermal product $\beta$ for the substrate material, and the expression $C$. In Eq. (5), the variable $C$ is specified in terms of the surface heat transfer coefficient, $c_{h}$, the model wall enthalpy, $h_{w}$, and the model wall temperature, $T_{w}$. Equation (6) shows $\beta$ is defined in terms of the thermal conductivity, $k$, and the thermal diffusivity, $\kappa$, of the model substrate material (either fused silica or Macor ${ }^{\mathbb{B}}$ ). The expression for $D$ in Eq. (7) contains the following variables: the adiabatic wall enthalpy, $h_{a w}$, the model wall enthalpy, $h_{w}$, the model wall temperature, $T_{w}$, and the initial temperature of the model surface, $T_{\text {init }}$.

$$
\begin{gathered}
\theta_{w}=T_{w}-T_{i n i t} \\
\Lambda=C \sqrt{t} / \beta \\
C=c_{h}\left(h_{w} / T_{w}\right) \\
\beta=k / \sqrt{\kappa} \\
D=h_{a w}\left(T_{w} / h_{w}\right)-T_{\text {init }}
\end{gathered}
$$

The Fay-Riddel1 ${ }^{22}$ heat transfer value is calculated at the stagnation point for a user-specified hemispherical nose radius. This heat transfer value is used as a reference value to derive nondimensional data from the convective heat transfer coefficients computed at the surface of the wind tunnel models. The images in Fig. 1 show a hemispherical model used to ensure the phosphor batch is behaving normally and the phosphor mix calibration is accurate. The pre-run temperature and run temperature images are aligned and the heat transfer coefficients at each image pixel that lies on the model are computed. At the stagnation point, the value of the nondimensional $h / h_{F-R}$ heat transfer is approximately equal to 1 , which confirms the phosphor system is properly calibrated for a given phosphor mix since the measured heat transfer is similar to the analytical value. Additional assumptions and equations in the IHEAT program are described in Refs. 1 and 2. 


\section{Pre-Run Temperature}

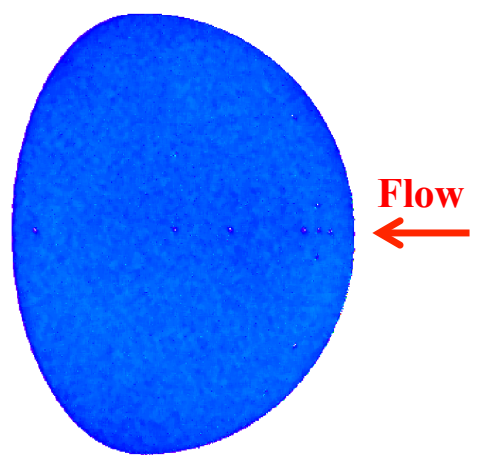

Run Temperature

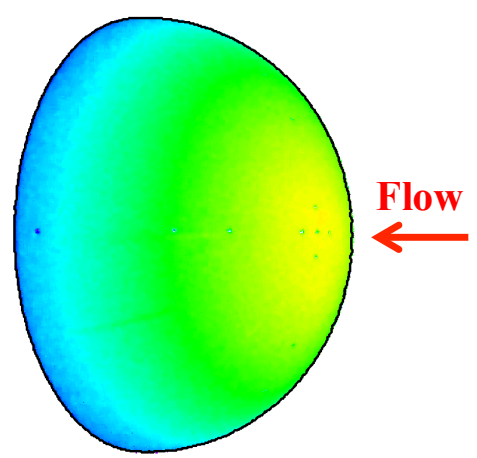

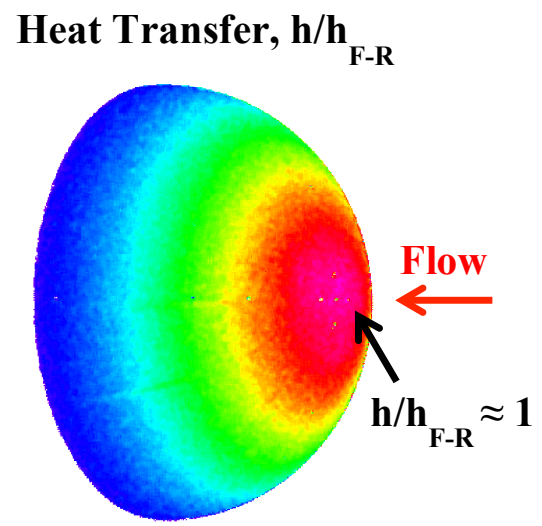

Figure 1. Hemisphere temperature and heat transfer images computed in IHEAT 4.0 (flow right to left).

IHEAT 4.0 contains numerous new or updated capabilities designed to streamline and increase the ease of use of the phosphor thermography data analysis process. Of these new or modified tools, primarily these four main categories will be discussed: diagnostic tools to check the quality of the acquired data, Piecewise and Auto Profile data extraction tools, batch processing techniques such as the Batch and Load Run tools, and a graphical uncertainty analysis tool.

\section{Capabilities of the IHEAT 4.0 Phosphor Thermography Data Reduction Program}

Global data from images of fluorescent fused-silica models are processed and analyzed in terms of temperature and heat transfer data in IHEAT 4.0. The legacy capability of the IHEAT 3.2 program is captured in IHEAT 4.0, as well as new features to speed up the data analysis process and to provide more information to the researcher during an aeroheating study in the LAL wind tunnels. One example of a legacy tool that is improved in IHEAT 4.0 is the ability to mask out erroneous data in the image that do not correspond to information on the wind tunnel model. The MATLAB $^{\circledR}$ version of this tool removes pixels that contain false data from the computed temperature, heat transfer and uncertainty images for a given run. These false signals can result from reflections of the UV light on the wind tunnel windows or phosphor coating fluorescence on the metal sting behind the ceramic model. Also, the boundary around the pre-run temperature image that allows the researcher to align the run temperature image to the pre-run temperature image, if a slight translation exists between the two sets of data, is updated to properly ignore the masked pixels off the body of the model.

\section{A. Diagnostic Tool}

One simple, but very useful, addition to the code is a three-part check of the pre-run temperature image that is automatically performed each time a pre-run image is loaded into the program. The output from this diagnostic tool is demonstrated in Figs. 2-4 using data from Space Shuttle models. This data set was acquired in the 20-Inch Mach 6 Tunnel to compare with Hypersonic Thermodynamic Infrared Measurements (HYTHIRM) data from a boundary layer transition flight experiment on the Space Shuttle. ${ }^{3}$ The temperatures on the windward surface of this model should be uniform and close to room temperature in the pre-run image taken under vacuum prior to flow through the tunnel.

This diagnostic tool compares the temperatures measured on the surface of the wind tunnel model to a thermocouple either on the leeward surface of the model, if available, or in the wind tunnel test section in three ways. The pre-run image and thermocouple temperatures are compared on a pixel level at each location on the model both (1) as an absolute difference displayed graphically in Fig. 2, and (2) in a histogram that quantitatively shows the distribution of the model temperatures relative to $\mathrm{a} \pm 10^{\circ} \mathrm{F}$ range surrounding the reference thermocouple temperature, as Fig. 3 demonstrates. To simplify these comparisons between the pre-run image temperatures and the thermocouple data, a third figure is generated that shows (3) pixels on the model as either green or red to indicate pixels either within or outside, respectively, of the $\pm 10^{\circ} \mathrm{F}$ span surrounding the thermocouple data. Thus, if the image of the model in this figure is mostly green (excluding the white pixels that lie off of the surface of the model, and any red pixels at the extreme edges and near fiducial marks on the model), then the pre-run temperatures are reasonably close to the reference value and uniform across the surface of the model. 


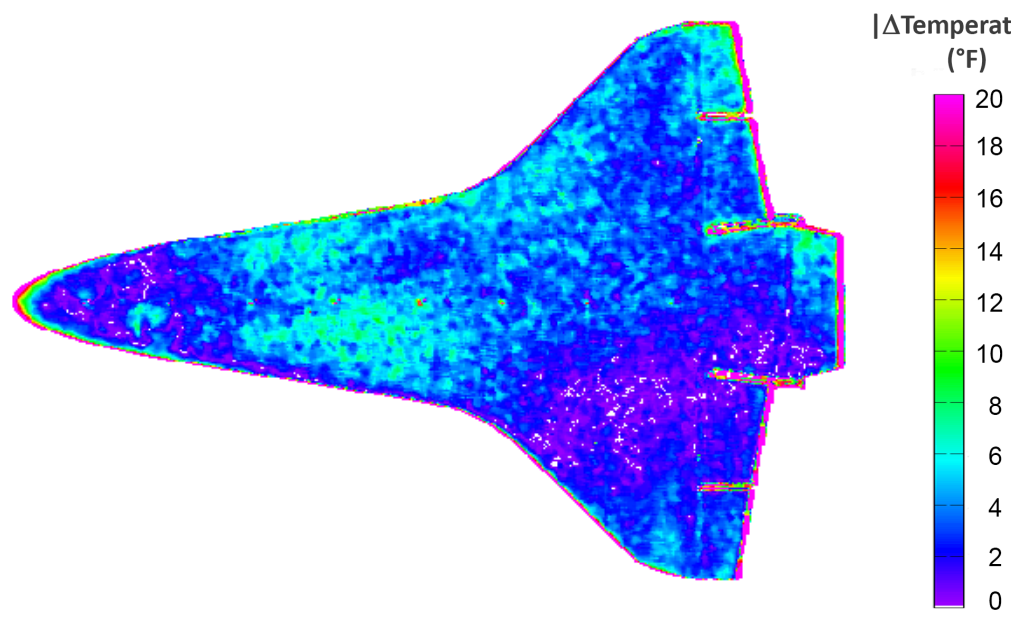

Figure 2. Absolute difference between pre-run image temperature values and initial tunnel test section temperature.

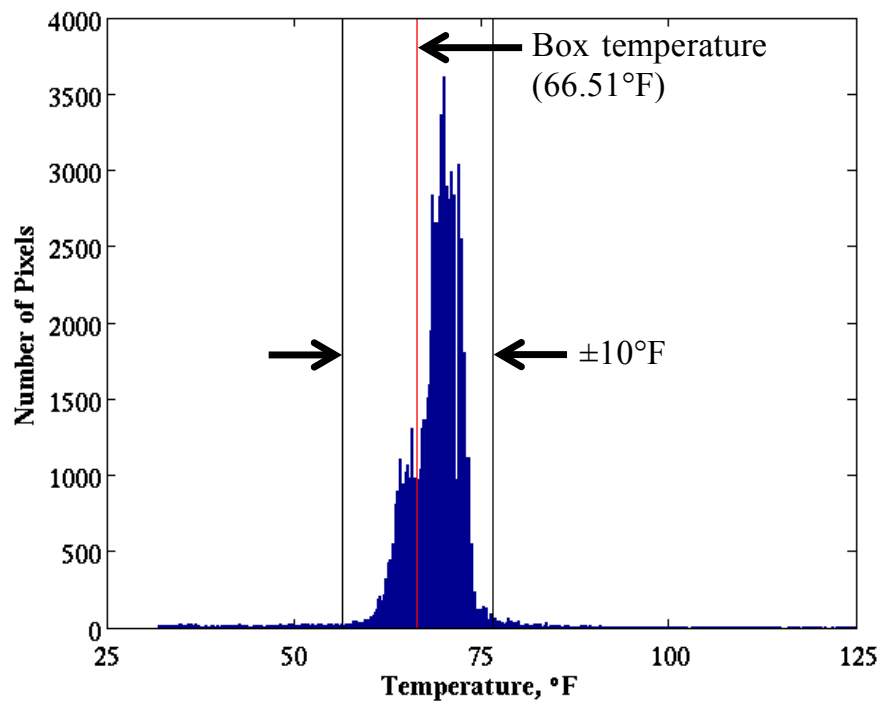

Figure 3. Check of pre-run temperature uniformity compared to the box temperature.

Green pixels are pre-run temperatures within $\pm 10^{\circ} \mathrm{F}$ of the initial run temperature in the tunnel.

Green $=$ Within Range

Red $=$ Out of Range

White $=$ Off the Model

Red pixels are temperatures outside of the $\pm 10^{\circ} \mathrm{F}$ range, usually near the edge of the model and at the fiducial marks.

White pixels in the image lie off of the model.
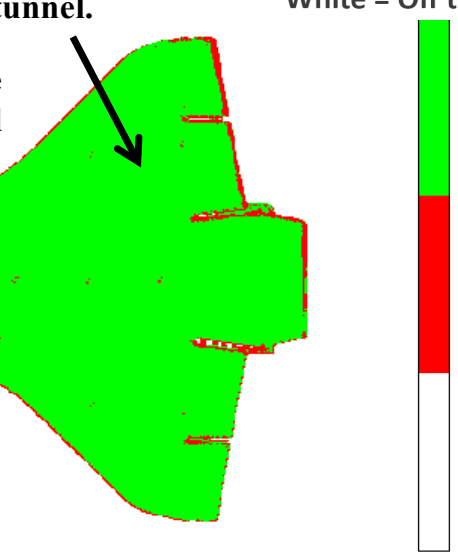

Figure 4. Color-coded image to quickly check the uniformity of the pre-run temperature data. 
This versatile diagnostic tool can be used to confirm that the calibration of the current phosphor mix is still valid, that the data acquisition system has been set-up properly, and that the temperatures measured by the reference thermocouple are accurate. Although other aspects of the overall system also could affect the output from the phosphor thermography program, verifying that the pre-run temperature image data are correct helps to ensure the quality of the final heat transfer values.

\section{B. Data Extraction Tools}

Both the legacy and the current versions of IHEAT contain Length and Profile tools that allow the user to extract data along a straight line from the 2D image of the model. The Length tool specifies a line cut that stretches from 0 to 1 in nondimensional length coordinates, while a Profile line cut normalizes the nondimensional positions along a straight line based on the length of the most recent Length line cut. A Piecewise line cut option has been added to IHEAT 4.0 to allow a user to create a segmented line cut that follows an interesting flow or geometry feature, such as a streamline or a leading edge, more easily than with multiple straight line cuts using the other tools. This line cut option is available both when individual images are analyzed and when the Batch function (described later) is used to process a series of images from a given run. The segmented Piecewise line cut, as well as the Length and Profile line cuts, can be automatically propagated to all of the time frames in a given run, eliminating the need to redefine the endpoints of the line segments in the cut each time, which saves valuable time in the analysis.

As an example of the Piecewise feature, a Piecewise line cut and a Length line cut were drawn on the image of the Hypersonic International Flight Research Experimentation (HIFiRE-1) $33^{\circ}$-flare model as shown in Fig. 5. The HIFiRE-1 data ${ }^{23}$ were used with permission. For this example, data were extracted from points 1 through 5 using the Piecewise tool and compared to the results obtained from a straight line cut between points 3 and 4 using the Length tool. The nondimensional heat transfer data match precisely in the flat region of the curve that represents the Length and the Piecewise results pulled from the line segment between points 3 and 4; however, the Piecewise tool also provides information about how the heat transfer values vary along the additional line segments defined by that tool. Although the segmented line is not overly complex in this example, the $2 \rightarrow 3$ and $4 \rightarrow 5$ segments are included to show short lines (i.e., a few pixels long) can be included to approximate a curved line to extract data from the image. The additional functionality of the Piecewise tool is especially useful when a model has a convex or concave surface, or if the temperature and heat transfer contours indicate a curved flow feature, both of which otherwise would be tedious to investigate using individual straight segments in a $2 \mathrm{D}$ image.

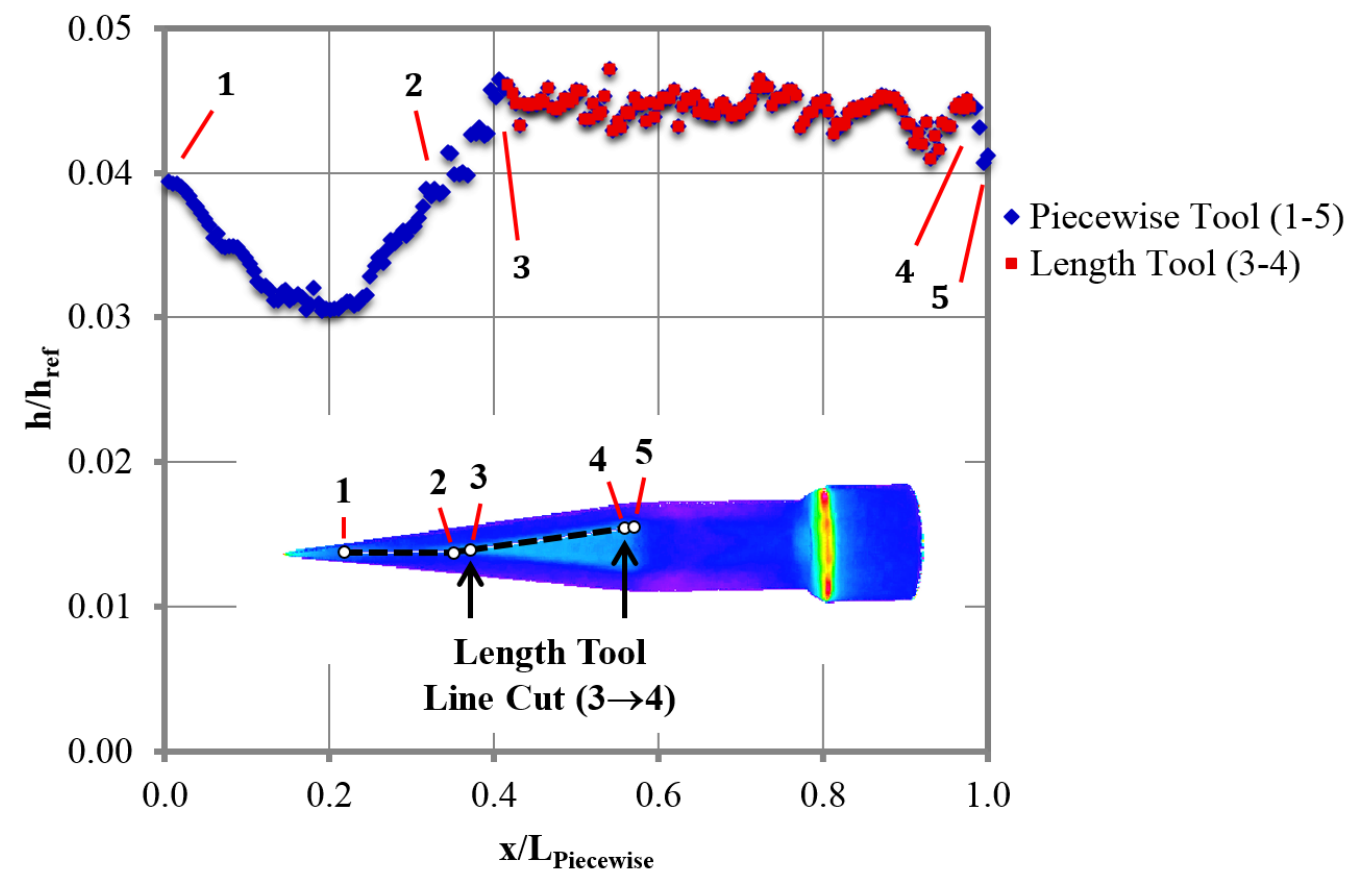

Figure 5. Example of Piecewise versus Length line cuts for a HIFiRE-1 heat transfer image in IHEAT 4.0. 
As the image in Fig. 6 shows, another tool in IHEAT (versions 3.2 and 4.0) called Auto Profile can be used to produce a Cartesian grid of perpendicular line cuts at specified $x / L$ and $y / y_{\text {ref }}$ locations, where $L$ is a characteristic length of the model, $y_{\text {ref }}$ is a characteristic dimension perpendicular to the length, and $x$ and $y$ are fractional distances of these characteristic dimensions. The locations of the fiducial marks on the model are provided as inputs to the Auto Profile tool, which are then used to determine the locations of the line cuts in the grid. The example grid in Fig. 6 includes axial lines at $x / L$ locations of 0.1 to 0.9 that are each $\Delta x / L=0.1$ apart, and longitudinal lines at $y / y_{\text {ref }}=0$ and \pm 0.25 . This grid is sparser in terms of $y / y_{\text {ref }}$ line cuts than the typical output from Auto Profile to simplify the diagram of the Auto Profile grid.

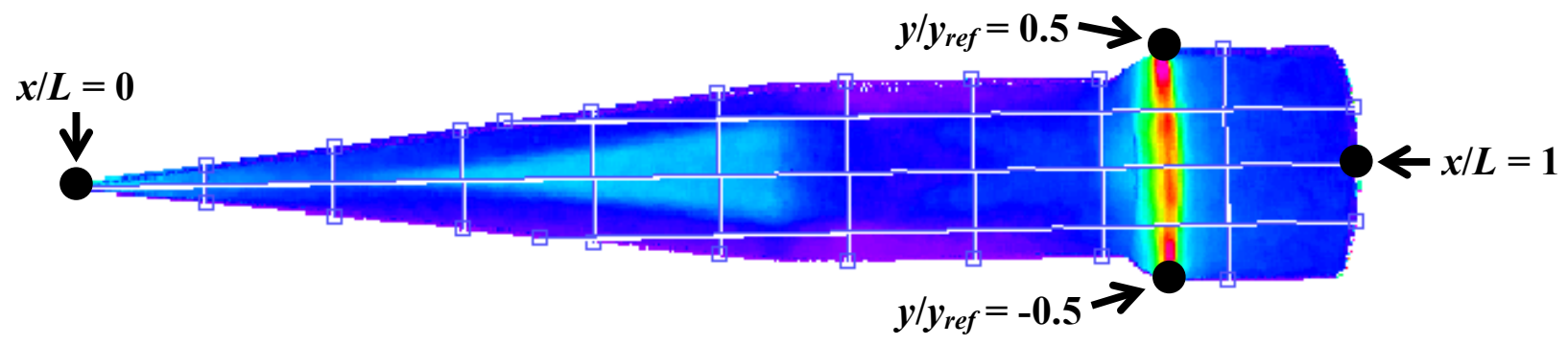

Figure 6. Auto Profile grid based on two $x / L$ and two $y / y_{\text {ref }}$ fiducial marks.

\section{Batch Processing Tools}

A new Batch tool in IHEAT 4.0 allows the user to process a single temporal frame manually and then automatically duplicate the process for a range of images from the same run (up to all of the images in the folder). The Batch process applies either an Auto Profile grid (as demonstrated in Fig. 6) or a Piecewise line cut, as specified by the researcher, to the series of run images. This tool automatically generates a graph of the temporal collapse of the centerline data (a necessary condition if the semi-infinite conduction assumption holds), or the comparison of either a characteristic line cut from the Auto Profile grid or the Piecewise line cut data from each image in the series over time during the run. The Batch tool loads in the appropriate input files and then computes the temperature and heat transfer data for the specified range of images. The tool then saves line cut data and IHEAT output files so that the researcher can access the data for any of the images individually.

The new Load Run option in IHEAT 4.0 requires less user interaction to load all of the images from a given run into the program, thus allowing more time for data analysis, rather than data processing. When a set of data is analyzed without the Load Run tool, the researcher must manually load multiple files into IHEAT 4.0 to compute heat transfer data. These files include a set-up file, a flow conditions file, a position gauge file, a "pre-run" temperature image (captured under vacuum prior to flow in the tunnel), and a "run" temperature image (captured at a specified time after the model reaches the wind tunnel centerline and is exposed to the flow). When the researcher selects the Load Run option, all of these files are loaded in automatically and drop-down menus are populated with the flow conditions files and run temperature images from the run directory. The researcher can select the corresponding files from the two drop-down menus and then compute the heat transfer for that instance during the run. The benefit of this tool, as with the Batch operation, is the automation that allows the user to analyze the data with fewer manual inputs to the software. Since IHEAT 4.0 is run remotely on a separate server, the researcher can complete either a Load Run or a Batch process simultaneously along with other tasks without reducing available memory on their host system, which equates to greater efficiency and more time savings during each test.

Both the Batch and Load Run functions speed up the data analysis process for multiple run temperature images from each aeroheating wind tunnel run. Nearly every step of the Batch process is automated (with the exception of an option for the researcher to reduce the number of images that are analyzed in the process), so the time required to analyze the data through this tool is reduced from hours to minutes for runs in which every frame of $30 \mathrm{~Hz}$ data is recorded. A figure showing the temporal collapse of the centerline data is generated automatically through the Batch tool. Thus, the researcher no longer has to (1) analyze a group of images individually, (2) copy the line cut data to a separate program, and (3) plot the data side by side manually, which saves time for other data analysis. The purpose of this plot is to determine the appropriate time frame during the run at which the vehicle's heating profile should be computed. In the example plot in Fig. 7, the line cut data changes as the model reaches the wind tunnel centerline (between frames 48 and 54, or about 1.6-1.8 seconds after model injection), and then collapses around frame 108, or about 2 seconds after the model reaches the wind tunnel centerline. Additional frames beyond the sample of seven shown in Fig. 7 are included in this plot using the Batch tool to better pinpoint the exact time of temporal collapse of the line cut data, which would be time and labor intensive without this automated feature. 


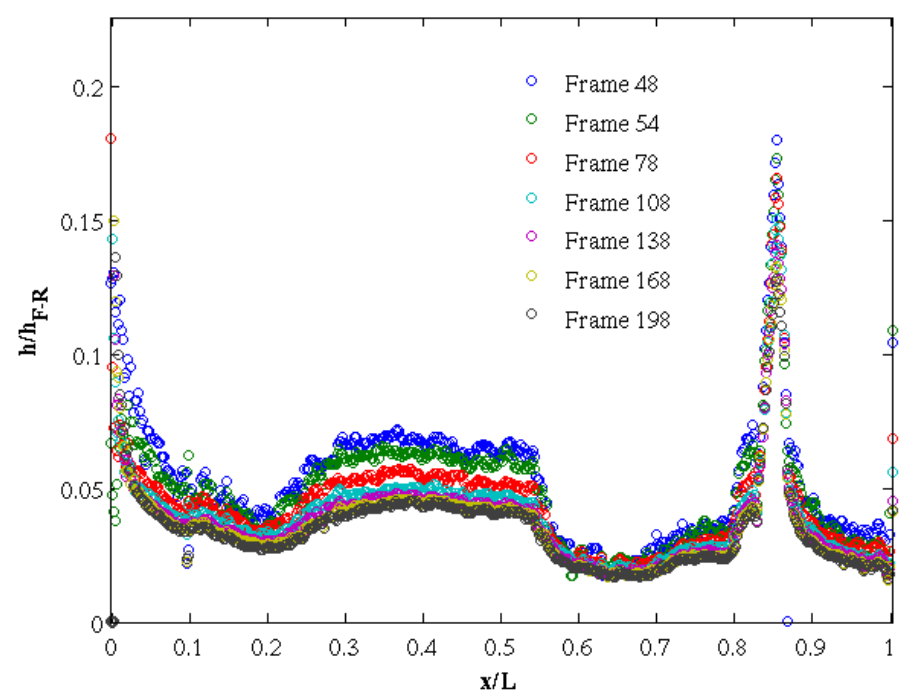

Figure 7. A plot showing temporal collapse using centerline data from a HIFiRE-1 model. ${ }^{23}$

\section{Uncertainty Analysis Tool}

Curve fits were previously developed for the 20-Inch Mach 6 and the 31-Inch Mach 10 Tunnels that account for the uncertainty in the computed heat transfer data due to the calibration of the phosphor mixture and the techniques applied to acquire and process the data. Errors in the heat transfer data based on the initial temperature, the run temperature, the adiabatic wall temperature, the substrate thermal properties, and the effective time (when the model reaches the wind tunnel centerline) are considered in the curve fits of the overall uncertainty, defined as the root of the sum of the uncertainties squared. More details about the types of errors included in the uncertainty curve fits are available in Refs. 1 and 2. In IHEAT 4.0, these uncertainty curve fits are applied to every image pixel that corresponds to a point on the model surface.

The Run Uncertainty image option in IHEAT 4.0 shows the level of confidence of each computed heat transfer coefficient. For the majority of the temperature range that the current phosphor system can measure, the total phosphor uncertainty varies between $7 \%$ and $10 \%$. In regions of less extreme temperature rise $\left(\Delta T \leq 25^{\circ} \mathrm{F}\right)$, the uncertainties are on the order of $\pm 25 \%$ or more. As the temperature difference between the pre-run temperature image and the run temperature image decreases, the uncertainty associated with the computed heat transfer value at that pixel in the image increases rapidly. Also, the level of uncertainty is greater for the data points along the edge of the model that cannot be lit properly, since the computed $\Delta T$ at these points is small. These trends in the uncertainty associated with each data pixel on the model surface are demonstrated in the Space Shuttle ${ }^{3}$ image in Fig. 8.

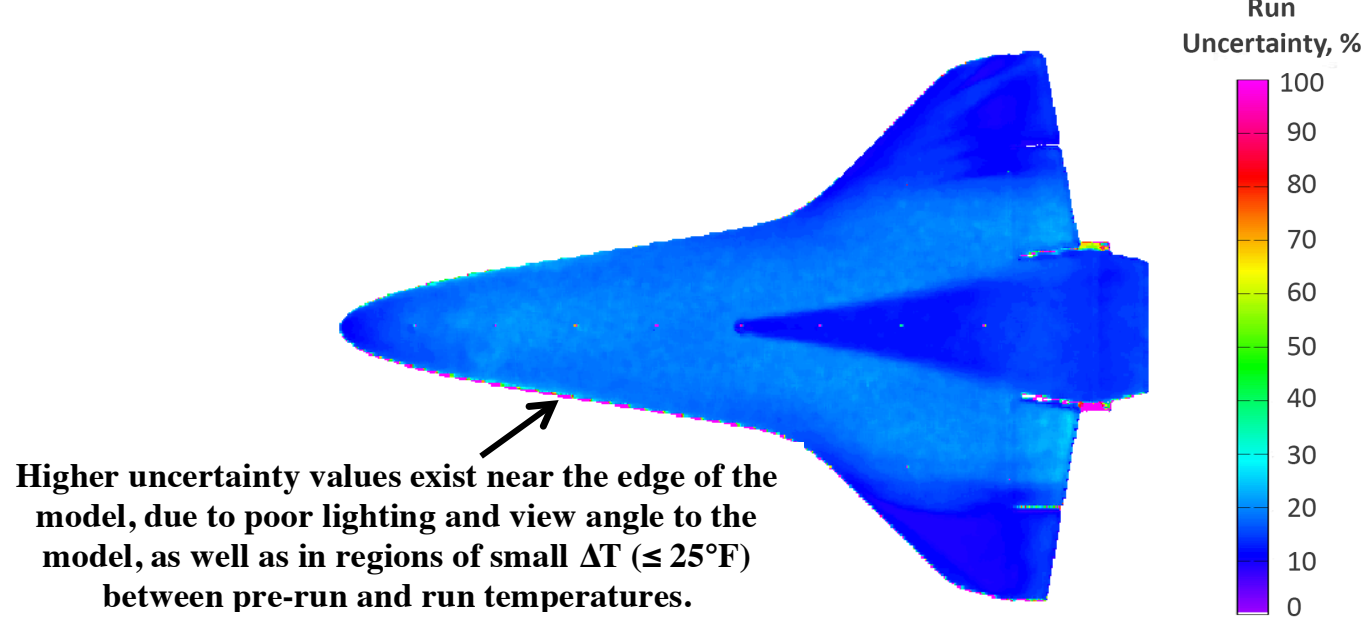

Figure 8. Uncertainty percentages in the heat transfer coefficients at each pixel on the Space Shuttle model. 


\section{Validation of IHEAT 4.0 Using IHEAT 3.2 Data}

A comparison of the results from the IHEAT 3.2 (written in PV-WAVE ${ }^{\mathbb{B}}$ ) and the IHEAT 4.0 (written in MATLAB ${ }^{\circledR}$ ) programs revealed very little difference between the outputs from the two programs except when certain filters were applied to the data. These additional filters in the legacy software were due to image resizing commands that were implemented to work within computer memory constraints that are no longer relevant. Therefore, these unintentional filters were deemed unnecessary and removed from the IHEAT 4.0 version of the program. Only a median filter with a 5 x 5 pixel neighborhood and a Sobel filter were retained. The median filter removes salt-and-pepper noise from the temperature data, and the Sobel filter enhances the edges of the models in the images (which otherwise are blurred by the median filter).

The images in Figs. 9-12 show the comparison between output data from IHEAT 3.2 and IHEAT 4.0 for models in the 20-Inch Mach 6 and the 31-Inch Mach 10 facilities, respectively. The left image in the first three figures corresponds to the absolute difference in the nondimensional heat transfer data from the two programs when both the median and the Sobel filters in each software package is applied. The right images show the difference between the data when neither a median nor a Sobel filter is applied. In Fig. 12, the images show the percent difference between the heat transfer data from the two programs, both with (right) and without (left) applied filters. Figures 9 and 10 provide results for a calibration hemisphere model in the two facilities, while Figs. 11 and 12 show the heat transfer differences on a Shuttle model ${ }^{3}$ in the 20-Inch Mach 6 Tunnel.

The similarities between the images in the first three figures indicate the differences between the IHEAT 3.2 and IHEAT 4.0 programs are independent of the model configuration. In those figures, the color bar limits range from 0 to 0.0001 in order to visualize the approximate magnitude of the heat transfer difference at each pixel on the model. The absolute differences in heat transfer in the regions where either no filters or only a median filter is applied are too small to show up in an image with a color bar scale from 0 to 1 . The limits on the color bar scale for the Shuttle percent difference data in Fig. 12 are also small, ranging from $0 \%$ to $0.01 \%$.

Over the majority of the model surface in each case, the differences between the nondimensional heat transfer data output from the two programs is small (on the order of $10^{-5}$ to $10^{-7}$ in terms of absolute differences, or less than $0.002 \%$ in the example of the Shuttle data). The Sobel filters employed by PV-WAVE ${ }^{\circledR}$ and MATLAB ${ }^{\circledR}$ differ enough to create significant differences between the IHEAT 3.2 and IHEAT 4.0 heat transfer outputs at the edge of the model and at fiducial mark locations on the model (represented by the pink pixels on the image). Since the pixels in these regions do not yield useful heat transfer data, and the Sobel filter in both programs achieves the goal of sharpening the edges that are inherently blurred due to the median filter, the Sobel filter applied in IHEAT 4.0 was deemed satisfactory. Compounding round-off errors due to slight differences in how data are stored and accessed in PV-WAVE ${ }^{\circledR}$ and MATLAB ${ }^{\circledR}$ could contribute to the small discrepancies in the data that exist elsewhere in the images whether a median filter is applied or no filters are applied to the data. The similarities between the filtered and unfiltered images in each figure indicate the median filter has a nearly identical effect in both programs.

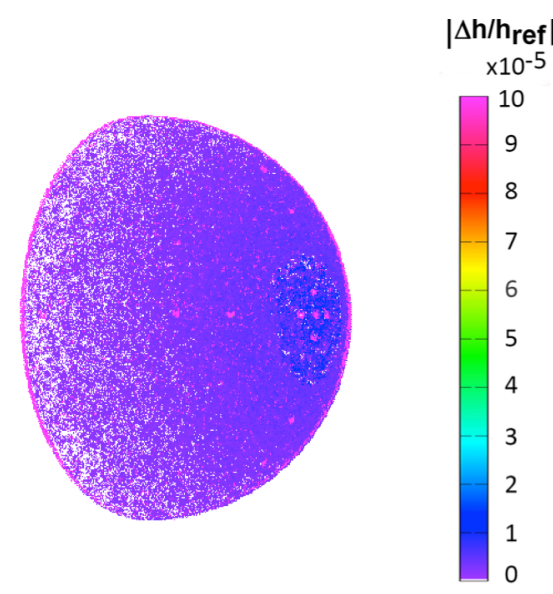

a) Sobel and median filters applied

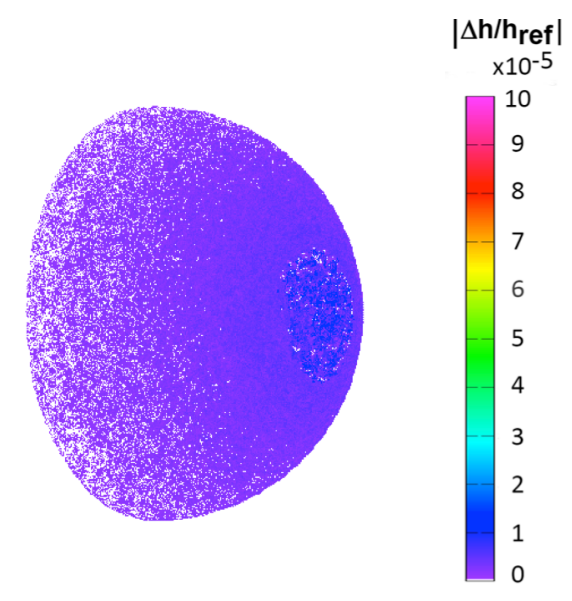

b) No filters applied

Figure 9. Absolute difference between IHEAT 4.0 and IHEAT 3.2 calibration hemisphere heat transfer data (20-Inch Mach 6 Tunnel). 


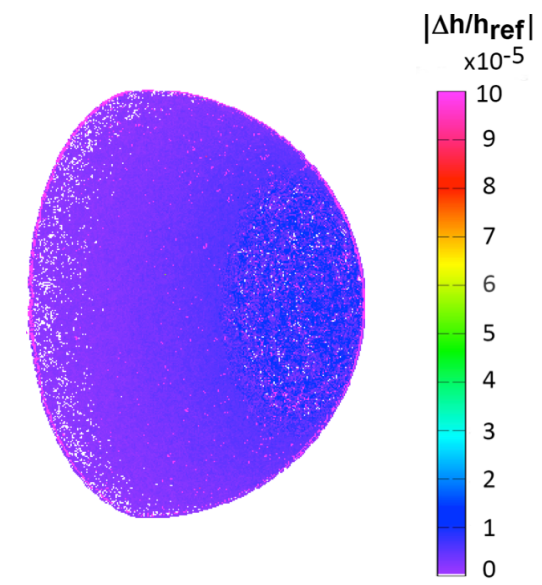

a) Sobel and median filters applied

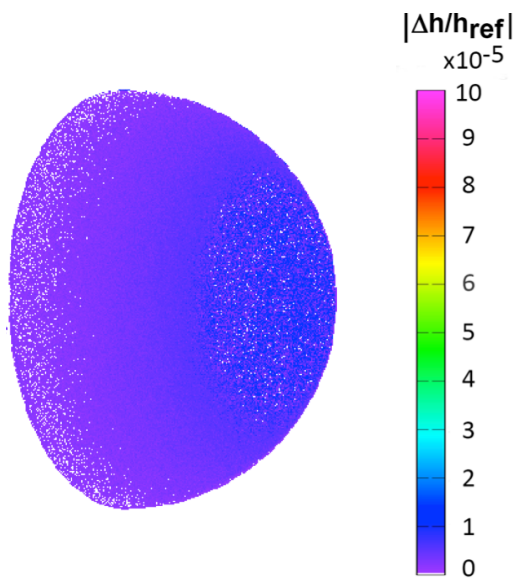

b) No filters applied

Figure 10. Absolute difference between IHEAT 4.0 and IHEAT 3.2 calibration hemisphere heat transfer data (31-Inch Mach 10 Tunnel).

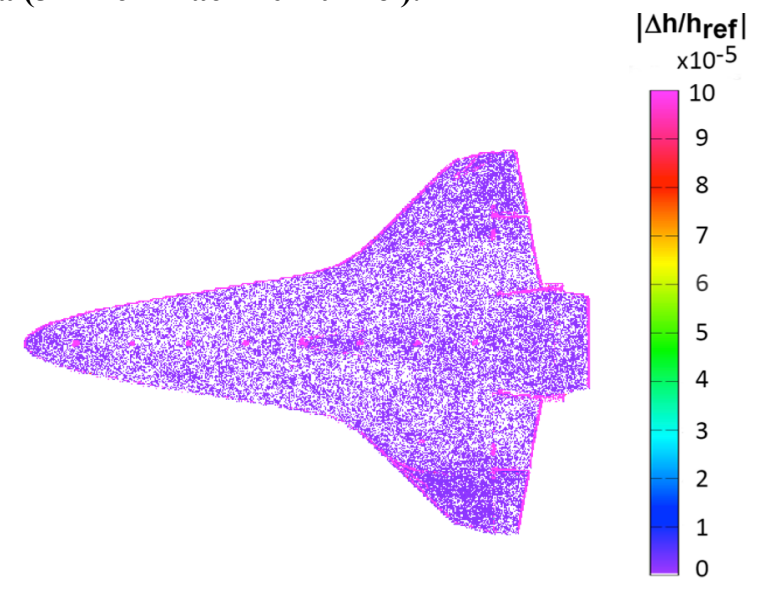

a) Sobel and median filters applied

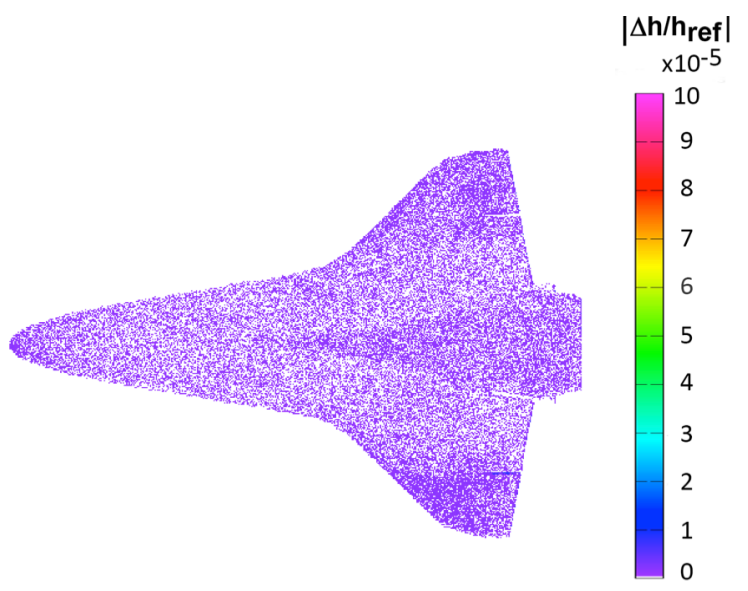

b) No filters applied

Figure 11. Absolute difference between IHEAT 4.0 and IHEAT 3.2 Space Shuttle heat transfer data (20Inch Mach 6 Tunnel).

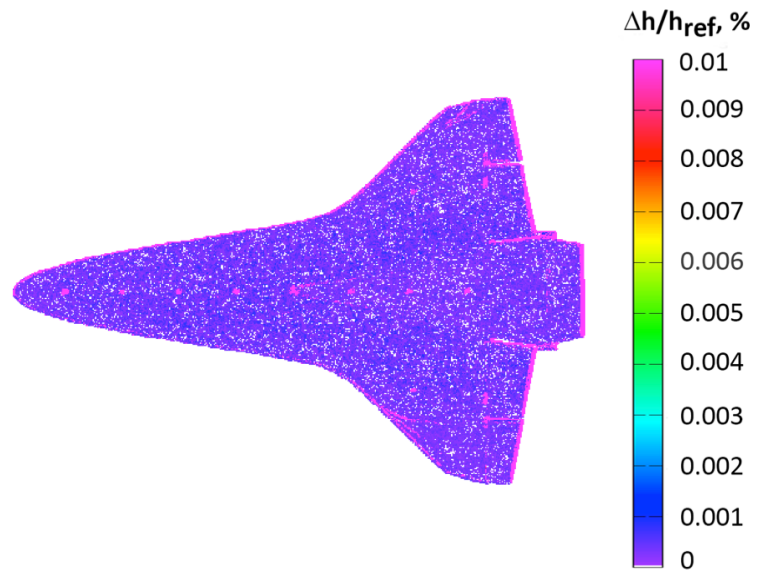

a) Sobel and median filters applied

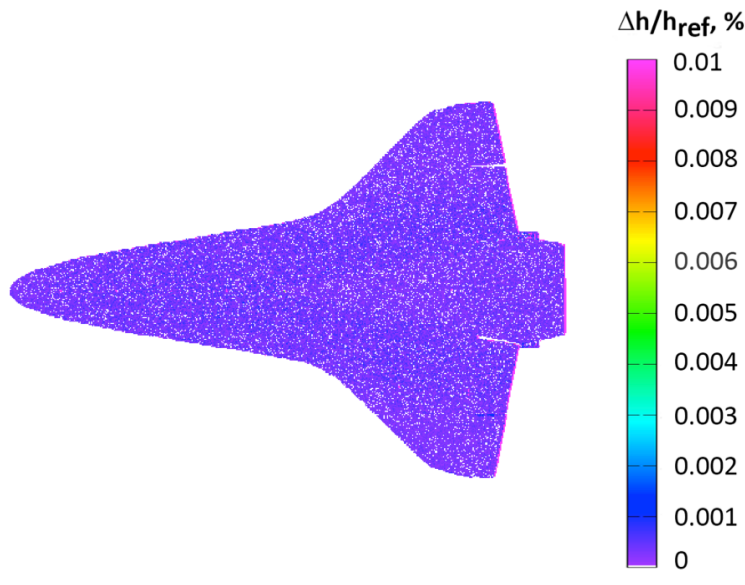

b) No filters applied

Figure 12. Percent difference between IHEAT 4.0 and IHEAT 3.2 Space Shuttle heat transfer data (20Inch Mach 6 Tunnel). 


\section{Ongoing Program Development}

Additional features will be included in future versions of the IHEAT program, such as functions to map the 2D images onto a 3D representation of the wind tunnel model, to extrapolate wind tunnel temperatures and heat transfer coefficients to approximate flight conditions, and to consider multidimensional conduction instead of assuming 1D conduction. A 3D Mapping algorithm developed in $\mathrm{C}++$ has been incorporated directly into IHEAT 4.0 to produce a Tecplot-formatted output mapped to a computer-aided design (CAD) model of the wind tunnel model, as shown in Fig. 13. This mapping module is currently being validated. Previously, this capability of mapping 2D heat transfer data to 3D CAD models was slow and tedious and required several iterations of the perspective and focal length parameters required to accurately map the data. The process is more automated in the 3D mapping tool in IHEAT 4.0 , and the researcher can continue to work with $2 \mathrm{D}$ data from the same or a different run in IHEAT 4.0 while the tool runs in the background to generate the mapped Tecplot output. As soon as the mapped data from this tool is validated, this IHEAT 4.0 module will be available for production use.

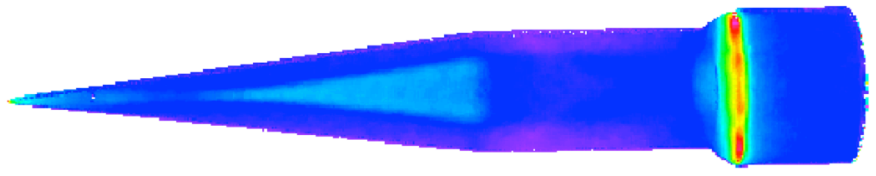

a) IHEAT 4.0 2D heat transfer image

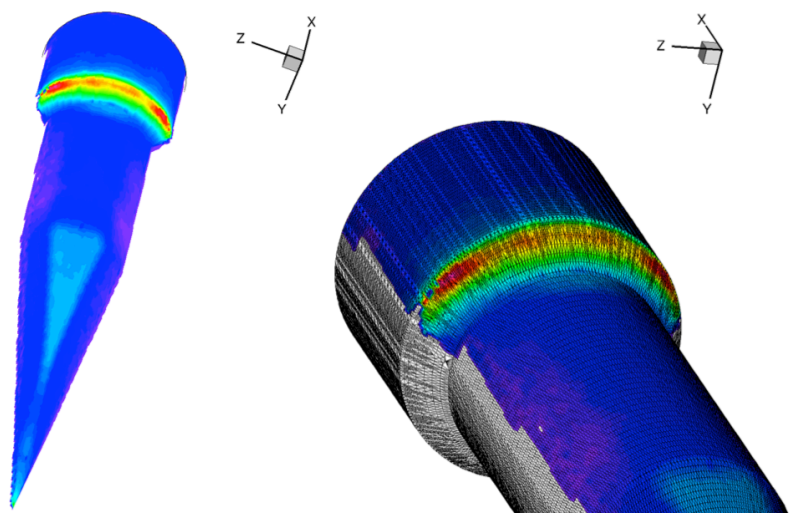

b) Two views of heat transfer data mapped to 3D CAD model

Figure 13. Mapped nondimensional heat transfer data for a HIFiRE-1 ${ }^{23}$ model.

An Extrapolation-to-Flight algorithm currently is being developed in IHEAT 4.0. This tool will compute the radiative equilibrium temperature and heat transfer coefficients that correspond to the predicted flight conditions for a given configuration using the wind tunnel data obtained for Mach 6 or Mach 10 flow at a similar angle of attack. A similar tool was previously used in IHEAT 3.2 on a case-by-case basis, but certain parameters were hard-wired and were changed manually to obtain extrapolation-to-flight data for different vehicle configurations. The algorithm for this tool in IHEAT 4.0 will be modified to permit the researcher to set the flight-vehicle-to-wind-tunnel-model ratios appropriately to apply the tool to new configurations without changing the base program. A prototype of this module has been added to IHEAT, but further testing and modifications are necessary to prepare this tool for inclusion in the production version of the program.

Multidimensional heat conduction analyses will be implemented in IHEAT 4.0 after the preceding features are fully operational. The 1D conduction assumption is typically sufficient for ceramic models tested in the LAL facilities during short wind tunnel runs, except on certain thin features such as leading edges and control surfaces and in regions with sharp temperature gradients. Including lateral conduction effects in the IHEAT 4.0 analysis would enable researchers to query data in these regions with increased confidence in the accuracy of the computed heat transfer data. The methodology is available for this program enhancement, but this capability currently has not been incorporated into IHEAT 4.0.

\section{Conclusion}

The IHEAT 4.0 phosphor thermography data analysis program is more efficient, more functional and more robust than the legacy IHEAT 3.2 program. Several features of the aeroheating data analysis process have been 
automated to reduce the required user interactions to compute heat transfer data for either one or all of the temperature images acquired during a wind tunnel run. By utilizing the program on a Unix server, rather than on the researcher's system, the program can perform Batch or other memory-intensive operations while the researcher completes other tasks without noticeably slowing down the host system. New features in IHEAT 4.0 improve the usefulness of the program, such as the diagnostic tool to check the uniformity and relative magnitude of pre-run image temperature measurements, the Piecewise tool that offers the user the ability to extract and plot data from nonlinear features in the data, the built-in check of the temporal collapse of run data in the new Batch feature, and the capability to easily duplicate line cuts produced in the Length, Profile, Piecewise or Auto Profile tools for other images in the run. Transferring the software to MATLAB ${ }^{\circledR}$ syntax from the PV-WAVE ${ }^{\circledR}$ programming language increased the stability of the software since the program is compiled as a stand-alone executable file that is not affected by changes to the base functions in new MATLAB ${ }^{\circledR}$ releases. Although IHEAT 3.2 employed additional filters due to image resizing commands that could not be exactly replicated in IHEAT 4.0, these filters were deemed unnecessary and were removed from IHEAT 4.0. The absolute differences between the heat transfer results from IHEAT 3.2 and 4.0 (excluding the effects of the unintentional filters) were on the order of $10^{-5}$ to $10^{-7}$, indicating the algorithm for computing heat transfer data is similarly implemented in the two programs that were written in different programming languages.

\section{Acknowledgments}

The authors would like to thank Ron Merski for developing the original IHEAT algorithm and assisting in the conversion from the legacy program to a MATLAB ${ }^{\circledR}$ executable file. The authors recognize Hui Fan's contribution to the program by implementing the $3 \mathrm{D}$ mapping algorithm in $\mathrm{C}++$. The authors also thank Karen Berger, Scott Berry, Kevin Hollingsworth, Brian Hollis, and Sheila Wright for their assistance in developing and debugging the program.

\section{References}

${ }^{1}$ Merski, N. R., "Reduction and Analysis of Phosphor Thermography Data with the IHEAT Software Package,” Reno, NV, 1997, AIAA Paper 1998-0712.

${ }^{2}$ Merski, N. R., "Global Aeroheating Wind-Tunnel Measurements Using Improved Two-Color Phosphor Thermography Method," Journal of Spacecraft and Rockets, Vol. 36, No. 2, 1999, pp. 160-170.

3 Berger, K. T., Hollingsworth, K. E., Wright, S. A., and Rufer, S. J., "NASA Langley Aerothermodynamics Laboratory: Hypersonic Testing Capabilities," Kissimmee, FL, 2015, AIAA Paper 20151337.

4 Voland, R., Huebner, L., and McClinton, C., "X-43A Hypersonic Vehicle Technology Development," IAC-05- D2.6.01, October 2005.

5 Berry, S., Auslender, A., Dilley, A., and Calleja, J., "Hypersonic Boundary-Layer Trip Development for Hyper- X," AIAA Paper 200-4012, August 2000.

${ }^{6}$ Hollis, B., Horvath, T., Berry, S., Hamilton, H., Thompson, R., and Alter, S., "X-33 Computational Aeroheating Predictions and Comparisons with Experimental Data," Journal of Spacecraft and Rockets, Vol. 38, 2001, pp. 658-669.

7 Sullivan, R., and Winters, B., "X-34 Program Overview,” July 1998, AIAA Paper 1998-3516.

8 Berry, S., Horvath, T., DiFulvio, M., Glass, C., and Merski, N., "X-34 Experimental Aeroheating at Mach 6 and Mach 10," January 1998, AIAA Paper 1998-881.

9 Machin, R., Stein, J., and Muratore, J., "An Overview of the X-38 Prototype Crew Return Vehicle Development and Test Program,” June 1999, AIAA Paper 1999-1703.

${ }^{10}$ Horvath, T., Berry, S., Merski, N. R., and Fitzgerald, S., "X-38 Experimental Aerothermodynamics," Journal of Spacecraft and Rockets, Vol. 41, 2004, pp. 272-292.

${ }^{11}$ Horvath, T., "Experimental Aerothermodynamics in Support of the Columbia Accident Investigation," January 2004, AIAA Paper 2004-1387.

${ }^{12}$ Horvath, T., Berry, S., Merski, N., Berger, K., Buck, G., Leichty, D., and Schneider, S., "Shuttle Damage/Repair from the Perspective of Hypersonic Boundary Layer Transition - Experimental Results," June 2006, AIAA Paper 2006-2919.

${ }^{13}$ Micol, J. R., "Langley Aerothermodynamics Facilities Complex: Enhancements and Testing Capabilities," January 1998, AIAA Paper 98-0147. 
${ }^{14}$ McGinley, C., Berry, S., Kinder, G., Barnwell, M., Wang, K., and Kirk, B., "Review of Orbiter Flight Boundary Layer Transition Data," June 2006, AIAA Paper 2006-2921.

${ }^{15}$ Everhart, J., "Supersonic/Hypersonic Laminar Heating Correlations for Rectangular and Impact Induced Open and Closed Cavities," Journal of Spacecraft and Rockets, Vol. 46, 2009, pp. 545-560.

${ }^{16}$ Norris, S., and Price, L., "Orion Program Status," September 2009, AIAA Paper 2009-6516.

${ }^{17}$ Berger, K., "Aerothermodynamic Testing of the Crew Exploration Vehicle at Mach 6 and Mach 10," Journal of Spacecraft and Rockets, Vol., No. 4, 2009, pp. 758-765.

${ }^{18}$ Lockwood, M., "Introduction: Mars Science Laboratory: The Next Generation of Mars Landers," Journal of Spacecraft and Rockets, Vol. 43, 2006, p. 257.

${ }^{19}$ Hollis, B., "Blunt-Body Entry Vehicle Aerothermodynamics: Transition and Turbulence on the CEV and MSL Configurations," June 2010, AIAA Paper 2010-4984.

${ }^{20}$ Hughes, S., Cheatwood, F., Dillman, R., Calomino, A., Wright, H., and DelCorso, J., "Hypersonic inflatable Aerodynamic Decelerator (HIAD) Technology Development Overview," May 2011, AIAA Paper 2011-2524.

${ }^{21}$ Jones, M. L., "Experimental Investigation of Shock-Shock Interactions over a 2-D Wedge at M =6," Virginia Polytechnic Instititute and State University, Hampton, VA, 2013, Master's Thesis.

${ }_{22}$ Fay, J. A., and Riddell, F. R., "Theory of Stagnation Point Heat Transfer in Dissociated Air," Journal of the Aeronautical Sciences, Vol. 25, No. 2, 1958, pp. 73-85, 121.

${ }^{23}$ Berger, K. T., Greene, F. A., and Kimmel, R., "Aerothermodynamic Testing and Boundary Layer Trip Sizing of the HIFiRE Flight 1 Vehicle,” Reno, NV, 2008, AIAA Paper 2008-640. 